\title{
Portion control for the treatment of obesity in the primary care setting
}

\author{
Rebecca L Kesman ${ }^{1}$, Jon O Ebbert ${ }^{1 *}$, Katherine I Harris ${ }^{2}$ and Darrell R Schroeder ${ }^{1}$
}

\begin{abstract}
Background: The increasing prevalence of obesity is a significant health threat and a major public health challenge. A critical need exists to develop and evaluate practical methods for the treatment of obesity in the clinical setting. One of the factors contributing to the obesity epidemic is food portion sizes. Limited data are available on the efficacy of visual or tactile devices designed to enhance patient understanding and control of portion sizes. A portion control plate is a commercially-available product that can provide visual cues of portion size and potentially contribute to weight loss by enhancing portion size control among obese patients. This tool holds promise as a useful adjunct to dietary counseling. Our objective was to evaluate a portion control intervention including dietary counseling and a portion control plate to facilitate weight loss among obese patients in a primary care practice.
\end{abstract}

Findings: We randomized 65 obese patients [body mass index (BMI) $\geq 30$ and $<40$ ] to an intervention including counseling by a dietitian incorporating a portion control plate or to usual care. Following initial consultation, patients in the intervention arm were contacted at 1,3, and 5 months by the dietician for brief follow-up counseling. Usual care subjects received instructional handouts on diet and exercise. Forty-two (65\%) subjects returned to have weight assessed at 6 months. Subjects in the portion control intervention had a greater percentage change ( \pm SD) in weight from baseline at 3 months $(-2.4 \% \pm 3.7 \%$ vs. $-0.5 \% \pm 2.2 \% ; p=0.041)$ and a non significant trend in weight change from baseline at 6 months $(-2.1 \% \pm 3.8 \%$ vs. $-0.7 \% \pm 3.7 \% ; p=0.232)$ compared with usual care. Nearly one-half of patients assigned to the portion control intervention who completed the study reported the overall intervention was helpful and the majority would recommend it to others.

Conclusions: Our findings suggest that a portion control intervention incorporating dietary counseling and a portion control plate may be effective for enhancing weight loss among obese subjects. A portion control intervention deserves further evaluation as a weight control strategy in the primary care setting.

Trial registration: Current controlled trials NCT01451554

\section{Background}

Since 1960, the prevalence of obesity has doubled in the United States and one-third of the adult population is obese (body mass index [BMI] $\geq 30.0$ ) [1]. Obesity is associated with significant morbidity, mortality, and healthcare costs and is a leading preventable cause of death [2]. Among individuals younger than 70 years of age, overweight-obesity is estimated to be the second leading cause of preventable death [3]. Pharmacologic interventions for the treatment of obesity are limited and are associated with significant side effects [4].

\footnotetext{
* Correspondence: ebbert.jon@mayo.edu

1 Mayo Clinic, 200 1st Street SW, Rochester, MN 55905, USA

Full list of author information is available at the end of the article
}

Exercise and psychological interventions have been demonstrated to be effective for weight loss, especially when combined with dietary strategies $[5,6]$.

Since portion sizes have a significant influence on energy intake [7], a portion control intervention may be a useful strategy to enhance and promote weight loss among obese patients. Food portion control interventions employ clinical strategies for increasing the awareness of portion sizes or caloric content of food using visual cues or tools as a point of reference, such as a plate demonstrating appropriate portion sizes. A randomized controlled study in Canada observed that a portion control plate led to significant weight loss and decreases in hypoglycemic medications among obese
C Biomed Central

() 2011 Ebbert et al; licensee BioMed Central Ltd. This is an open access article distributed under the terms of the Creative Commons Attribution License (http://creativecommons.org/licenses/by/2.0), which permits unrestricted use, distribution, and reproduction in any medium, provided the original work is properly cited. 
patients with diabetes [8]. Another study among obese young people discovered that a portable computerized device weighing the meal plate and providing feedback to slow the rate of food intake and reduce total intake was effective for decreasing body weight [9].

To date, no studies have been published assessing the effectiveness of portion control interventions in a primary care setting. We conducted a pilot study to assess the effectiveness of an intervention including a portion control plate and dietary counseling for weight loss among obese patients in a general medicine primary care practice.

\section{Methods}

\section{Subjects}

The Mayo Foundation Institutional Review Board (IRB) reviewed and approved the study protocol prior to recruitment and enrollment. Potential subjects were recruited from a primary care internal medicine practice at the Mayo Clinic in Rochester, MN. Male and female patients were eligible for enrollment if they were men and women between the ages of 18 and 75 with a body mass index (BMI) of $\geq 30$ and $<40$ (obesity classes II and III). Exclusion criteria included presence of active cancer, participation in an organized weight loss program, current weight loss medication, history of bulimia or anorexia, current treatment for psychiatric illness other than anxiety or depression, surgery within the 3 months before enrollment or planned during the study period, current or planned pregnancy, and a history of gastric bypass or planned gastric bypass.

\section{Procedures}

Prior to the patient's appointment with their primary care provider, the patient's medical record was reviewed to assess BMI. Medical records were reviewed only if the patient had provided a universal research authorization to the Mayo Clinic allowing for chart reviews of this nature. If potentially eligible based on BMI, a letter was sent to the patient informing them of the study and asking them to discuss enrollment with their primary care provider at their upcoming appointment.

At the appointment, the patient was informed of the study and asked if they would like to participate. If the patient was interested, a study coordinator contacted the patient to discuss details of the study and confirm eligibility. Patients completed informed consent and were randomized. Randomization was stratified by gender using sealed envelopes. Patients were randomized to either the portion control intervention or usual care by the study coordinator.

If the patient was randomized to usual care, Mayo Clinic pamphlets entitled "Lifestyle Changes for Healthy Weight" and "Exercise: Getting Started and Staying
With It" were given to the patient. These patients received pamphlets in an attempt to standardize potentially heterogeneous dietary and exercise advice received from different primary care providers. If the patient was randomized to the portion control intervention, a 60minute appointment with a dietician was scheduled. At the dietitian appointment, a baseline assessment of weight history, nutritional intake, and exercise and non exercise activity was conducted. The dietician reviewed food choices, portion control, consistency and timing of meals, meal plans and appropriate use of snacks. The patient received specific written instructions on how to use a commercially-available calibrated portion control plate and bowl. The portion control plate was made of clear glass with black print dividing it into three sections (one one-half and two one-quarter sections). One-half of the plate was labeled "vegetables," one-quarter was labeled "fish, lean meat, chicken \& nuts," and one-quarter was labeled "potatoes, pasta, rice, beans and whole grains." The bowl was clear glass with black print designating " $1 / 3$ cup," " $1 / 2$ cup," and " 1 cup." The patient was instructed to use the plate for their largest meal of the day and encouraged to use the plate/bowl for all meals.

The patients were followed for 6 months following randomization. All patients were scheduled for weight assessments at 3 and 6 months. Weight assessments were conducted using digital clinic scales with shoes off and clothes worn. Patients in the portion control intervention group were contacted by phone or email (based on patient preference) at 1,3 , and 5 months by the dietitian who briefly provided additional counseling and assistance. At study completion, patients in the portion control group were provided with a survey to assess their satisfaction with the intervention.

\section{Statistical Methods}

The primary endpoint was the percentage weight change from baseline at 3 and 6 months. Analyses were performed using an intention-to-treat approach whereby subjects were analyzed according to randomized treatment. No covariate adjustment was included in the primary analysis. We used descriptive statistics to summarize demographic data and other baseline characteristics, along with results of the satisfaction survey.

Weight change from baseline at 3 and 6 months was expressed as a percentage of baseline weight. For this study, we anticipated that the difference in weight change between groups would likely be larger at 6 months than at 3 months (i.e. time-by-treatment interaction effect) and that the variance of the weight change would likely be larger at 6 months than at 3 months. For these reasons, we decided a priori that for this pilot study we would perform separate analyses for the 3 and 
6 month time points. Weight change was compared between groups using the two-sample t-test. We conducted analyses excluding subjects with missing data (complete case analysis) and also using the last observation carried forward (LOCF) to impute values for subjects who discontinued study participation. In all cases, the difference in weight change between groups (plate minus control) was summarized using a point-estimate and corresponding 95\% confidence interval. Two-tailed p-values $\leq 0.05$ were considered statistically significant.

The assumptions used for sample-size calculations were based upon previous literature [8]. Assuming a mean percentage of weight loss of $2 \%$ in the intervention group and $0 \%$ in the controls with a standard deviation of $3 \%$, we determined that a total sample-size of 80 patients would provide statistical power (two-tailed, alpha $=0.05$ ) of $83 \%$ to detect a difference between groups. Due to a shortage of portion control bowls which could not be reordered from the original source, the decision was made to discontinue enrollment after randomizing 65 subjects. Under the assumption that the standard deviation is $3 \%$, an effective sample-size of 65 subjects provided statistical power (two-tailed, alpha = 0.05 ) of $75 \%$ to detect a difference in weight change of $2 \%$ between groups.

\section{Results}

Sixty five patients (25 men and 40 women) were randomized. Groups were similar at baseline (Table 1). The overall mean \pm SD age was $55.9 \pm 10.0$ years with a mean weight of $98.2 \pm 12.6 \mathrm{kgs}$ (range 74.7 to 129.3 $\mathrm{kgs})$. Twenty-three percent of subjects $(\mathrm{N}=15)$ had diabetes and $5 \%(\mathrm{~N}=3)$ were current smokers.

Among those who completed the study, subjects in the portion control intervention had a greater percentage weight change from baseline compared to usual care $(-2.4 \% \pm 3.7 \%$ vs. $-0.5 \% \pm 2.2 \% ; \mathrm{p}=0.041)$ at 3 months (Table 2). A trend toward increased weight loss with the portion control intervention was observed using the LOCF analysis $(-1.7 \pm 3.3$ vs. $-0.4 \pm 1.9 ; \mathrm{p}=$ 0.062 ) at 3 months. Non significant trends toward increased weight loss with the portion control intervention were observed at six months (Table 2). Among participants in the portion control intervention, the percentage weight change from baseline to 6 months did not differ significantly between those who reported using the portion control plate $\geq 2$ times per day compared to those using the plate $<2$ times per day $(-2.7 \pm$ 3.8 vs $-1.1 \pm 3.8 ; \mathrm{p}=0.40)$.

Among the 19 patients in the intervention group who completed the study, $47 \%$ perceived that the overall portion control intervention was helpful. Sixty-eight percent endorsed that the counseling at the dietitian visit was helpful and $79 \%$ would recommend the
Table 1 Baseline subject characteristics

\begin{tabular}{|c|c|c|}
\hline & $\begin{array}{c}\text { Usual Care } \\
\mathrm{N}=32\end{array}$ & $\begin{array}{c}\text { Portion Control } \\
\qquad N=33\end{array}$ \\
\hline \multicolumn{3}{|l|}{ Age, years } \\
\hline Mean \pm SD & $56.3 \pm 10.7$ & $55.4 \pm 9.4$ \\
\hline Range & 32 to 75 & 31 to 74 \\
\hline \multicolumn{3}{|l|}{ Gender, N (\%) } \\
\hline Male & $12(38)$ & $13(39)$ \\
\hline Female & $20(62)$ & $20(61)$ \\
\hline \multicolumn{3}{|l|}{ Weight, kg } \\
\hline Mean \pm SD & $98.8 \pm 12.5$ & $97.6 \pm 12.8$ \\
\hline Range & 75.2 to 127.2 & 74.7 to 129.3 \\
\hline \multicolumn{3}{|l|}{ Education, N (\%) } \\
\hline High school graduate & $7(22)$ & $8(24)$ \\
\hline Some college & $12(38)$ & $14(42)$ \\
\hline 4-year college degree or more & $13(41)$ & $11(33)$ \\
\hline \multicolumn{3}{|l|}{ Work status, N (\%) } \\
\hline Full time & $19(59)$ & $22(67)$ \\
\hline Part time & $2(6)$ & $2(6)$ \\
\hline Unemployed & $1(3)$ & $1(3)$ \\
\hline Retired & $10(31)$ & $8(24)$ \\
\hline \multicolumn{3}{|l|}{ Current tobacco use, N (\%) } \\
\hline No & $30(94)$ & $32(97)$ \\
\hline Yes & $2(6)$ & $1(3)$ \\
\hline \multicolumn{3}{|l|}{ Diabetes, N (\%) } \\
\hline No & $25(78)$ & $25(76)$ \\
\hline Yes & $7(22)$ & $8(24)$ \\
\hline \multicolumn{3}{|l|}{ Current exercise, N (\%) } \\
\hline None & $4(12)$ & $4(12)$ \\
\hline 1 to 90 minutes per week & $8(25)$ & $13(39)$ \\
\hline 91 to 150 minutes per week & $16(50)$ & $9(27)$ \\
\hline$>150$ minutes per week & $4(12)$ & $7(21)$ \\
\hline
\end{tabular}

portion plate to family or friends. Thirty-two percent reported that they used the plate for one meal per day, while $37 \%$ and $26 \%$ said that they used the plate for two or three meals per day. Forty-two percent of patients said that they would continue to use the portion plate after the study.

\section{Discussion}

In this pilot study, we observed weight loss at three months among obese patients in a primary care practice who received a portion control intervention. Our findings are comparable to the amount of weight loss achieved in a study performed in Canada which observed a weight loss of $1.8 \% \pm 3.9 \%$ among obese diabetics using a portion control plate compared with $0.1 \%$ $\pm 3.0 \%$ among the control group. In the current study, patients in the usual care group receiving two Mayo Clinic designed educational handouts had essentially no weight loss. Almost half of the patients in our study group perceived the overall portion control intervention 
Table 2 Weight change from baseline at 3 and 6 months

\begin{tabular}{|c|c|c|c|c|c|c|c|}
\hline & \multicolumn{2}{|c|}{$\begin{array}{l}\text { Usual Care } \\
(\mathrm{N}=32)\end{array}$} & \multicolumn{2}{|c|}{$\begin{array}{l}\text { Portion Control } \\
\quad(\mathrm{N}=33)\end{array}$} & \multicolumn{2}{|c|}{$\begin{array}{l}\text { Difference in Means } \\
\text { (Plate-Control) }\end{array}$} & \multirow[b]{3}{*}{ P-value* } \\
\hline & & mean $\pm S D$ & & mean $\pm S D$ & & & \\
\hline & $\mathbf{N}$ & $(\min , \max )$ & $\mathbf{N}$ & $(\min , \max )$ & Estimate & (95\% C.I.) & \\
\hline \multicolumn{8}{|l|}{3 months } \\
\hline \multicolumn{8}{|l|}{ Change from baseline (kgs) } \\
\hline Complete case & 24 & $\begin{array}{l}-0.6 \pm 2.2 \\
(-6.4,+2.3)\end{array}$ & 23 & $\begin{array}{c}-2.2 \pm 3.6 \\
(-11.6,+4.2)\end{array}$ & -1.6 & $(-3.3,+0.1)$ & 0.071 \\
\hline Last value carried forward & 32 & $\begin{array}{l}-0.4 \pm 1.9 \\
(-6.4,+2.3)\end{array}$ & 33 & $\begin{array}{c}-1.5 \pm 3.1 \\
(-11.6,+4.2)\end{array}$ & -1.1 & $(-2.4,+0.2)$ & 0.099 \\
\hline \multicolumn{8}{|l|}{$\%$ change from baseline } \\
\hline Complete case & 24 & $\begin{array}{l}-0.5 \pm 2.2 \\
(-5.2,+2.3)\end{array}$ & 23 & $\begin{array}{c}-2.4 \pm 3.7 \\
(-11.4,+3.9)\end{array}$ & -1.8 & $(-3.6,+0.7)$ & 0.041 \\
\hline Last observation carried forward & 32 & $\begin{array}{l}-0.4 \pm 1.9 \\
(-5.2,+2.3)\end{array}$ & 33 & $\begin{array}{c}-1.7 \pm 3.3 \\
(-11.4,+3.9)\end{array}$ & -1.3 & $(-2.6,+0.1)$ & 0.062 \\
\hline \multicolumn{8}{|l|}{6 months } \\
\hline \multicolumn{8}{|l|}{ Change from baseline (kgs) } \\
\hline Complete case & 23 & $\begin{array}{c}-0.9 \pm 4.2 \\
(-15.7,+3.9)\end{array}$ & 19 & $\begin{array}{l}-1.9 \pm 3.7 \\
(-10.0,+6.1)\end{array}$ & -1.0 & $(-3.5,+1.5)$ & 0.414 \\
\hline Last value carried forward & 32 & $\begin{array}{c}-0.5 \pm 3.6 \\
(-15.7,+3.9)\end{array}$ & 33 & $\begin{array}{l}-1.0 \pm 3.0 \\
(-10.0,+6.1)\end{array}$ & -0.5 & $(-2.2,+1.1)$ & 0.528 \\
\hline \multicolumn{8}{|l|}{$\%$ change from baseline } \\
\hline Complete case & 23 & $\begin{array}{c}-0.7 \pm 3.7 \\
(-12.3,+3.7)\end{array}$ & 19 & $\begin{array}{l}-2.1 \pm 3.8 \\
(-9.8,+5.6)\end{array}$ & -1.4 & $(-3.7,+0.9)$ & 0.232 \\
\hline Last observation carried forward & 32 & $\begin{array}{l}-0.4 \pm 3.2 \\
(-12.3,+3.7)\end{array}$ & 33 & $\begin{array}{l}-1.2 \pm 3.1 \\
(-9.8,+5.6)\end{array}$ & -0.8 & $(-2.3,+0.8)$ & 0.311 \\
\hline
\end{tabular}

* Two-sample t-test

to be helpful and the majority would suggest the portion control plate to family and friends.

The attrition rate in the current study (35\%) at 6 months is higher than the mean attrition rate of $22 \%$ within 6 months in studies of behavioral interventions for obesity [10]. However, our attrition rate is significantly less than commercial weight loss programs with reported attrition rates as high as $70 \%$ at 12 weeks [11]. Our higher attrition may relate to the reduced patient interaction between three and six months. Indeed, our attrition rate at 3 months up to which time the dietician was more engaged with the portion control intervention group was $28 \%$ increasing to $35 \%$ by the end of the trial. Higher program adherence might be expected with a clinical trial involving more frequent contacts with interventionists.

Despite the fact that we had more patients in the portion control intervention drop-out, counseling by the dietitian was ranked favorably. The majority of subjects felt that the portion plate would be helpful for other people trying to lose weight and would recommend it to family members or friends interested in losing weight. However, if we assume that the drop-outs did not have a favorable response to the portion control plate then we should be less optimistic about how the plate will be perceived by obese patients in a general medical practice. Ideally, the portion control plate could be part of a menu of options that patients interested in losing weight could use. Favorable perceptions of a portion control plate could be enhanced by leveraging existing technologies and providing feedback about the speed of food consumption to enhance satiety with smaller portions [9].

Forty-two percent of patients reported that they would continue to use the portion control plate after the end of the study which seems high considering that most obese patients do not adhere to dietary programs beyond six months [12]. We hypothesize that the provision of a re-usable visual tool may increase long-term adherence to a clinical weight loss intervention. We did not conduct follow-up surveys to determine what percentage of subjects actually continued to use the portion control plate.

In June 2011, the United States Department of Agriculture replaced the food pyramid with the "food plate" [13]. The new image is a plate-shaped diagram or pie chart. This model could be leveraged to promote the use of a portion control plate in clinical practice. Interventions effectively linking the new "food plate" model to the portion control plate to promote healthy eating and weight loss are needed.

Limitations of our study include the small sample size, an inability to blind the intervention, and incomplete 
follow-up. In addition, we excluded morbidly obese patients and patients under current treatment for psychiatric illness other than anxiety or depression which limits the ability to generalize to the usual primary care practice. Our intervention included counseling by a dietitian, the portion control plate and bowl, and calls or emails by a dietitian. Our multifaceted intervention makes it difficult to determine the clinical effect of each individual component on weight loss. Future studies should include an additional study arm to evaluate the effect of the portion control plate with dietary counseling compared to dietary counseling alone.

Unfortunately, our study was too small to assess for gender, age effects or baseline BMI effects. Previous research has demonstrated that weight loss is more likely to occur among patients who are older, weigh less at baseline [14] and are male [12]. Future studies of a portion control intervention should evaluate these potential predictors of treatment response.

\section{Conclusions}

Effective and practical tools to combat the epidemic of obesity desperately need to be evaluated and disseminated. Our pilot study demonstrated weight loss among obese patients receiving a portion control intervention including dietary counseling and a portion control plate in a primary care general medicine practice. Larger studies are needed to assess the utility of portion control tools in primary care and corroborate the findings of our small clinical pilot.

\section{List of Abbreviations}

BMI: body mass index; SD: standard deviation; Cl: confidence interval.

\section{Acknowledgements and Funding}

This study was funded by the Mayo Clinic.

\section{Author details}

${ }^{1}$ Mayo Clinic, 200 1st Street SW, Rochester, MN 55905, USA. ${ }^{2}$ University of lowa Hospitals and Clinics; 200 Hawkins Drive, E 327-1 GH; lowa City, IA 52242, USA.

\section{Authors' contributions \\ RLK conceived of the study, wrote the protocol, obtained funding, coordinated subject enrollment, and drafted the manuscript. JOE assisted with study design and oversight and provided critical input on protocol design, data interpretation and manuscript writing. KIH assisted RLK and JOE with study design, study enrollment, data analysis and manuscript writing. DRS performed the randomization and conducted the data analyses. All authors have read and approved the final version.}

\section{Competing interests}

The authors declare that they have no competing interests.

Received: 22 March 2011 Accepted: 9 September 2011

Published: 9 September 2011

\section{References}

1. Flegal KM, Carroll MD, Ogden CL, Curtin LR: Prevalence and trends in obesity among US adults, 1999-2008. JAMA 303(3):235-241.
2. Clinical Guidelines on the Identification, Evaluation, and Treatment of Overweight and Obesity in Adults. [http://www.nhlbi.nih.gov/guidelines/ obesity/ob_gdlns.pdf].

3. Danaei G, Ding EL, Mozaffarian D, Taylor B, Rehm J, Murray CJ, Ezzati M: The preventable causes of death in the United States: comparative risk assessment of dietary, lifestyle, and metabolic risk factors. PLOS Med 2009, 6(4):e1000058.

4. Li Z, Maglione M, Tu W, Mojica W, Arterburn D, Shugarman LR, Hilton L, Suttorp M, Solomon V, Shekelle PG, et al: Meta-analysis: pharmacologic treatment of obesity. Ann Intern Med 2005, 142(7):532-546.

5. Shaw K, Gennat H, O'Rourke P, Del Mar C: Exercise for overweight or obesity. Cochrane Database Syst Rev 2006, , 4: CD003817.

6. Shaw K, O'Rourke P, Del Mar C, Kenardy J: Psychological interventions for overweight or obesity. Cochrane Database Syst Rev 2005, , 2: CD003818.

7. Rolls BJ, Morris EL, Roe LS: Portion size of food affects energy intake in normal-weight and overweight men and women. Am J Clin Nutr 2002, 76(6):1207-1213.

8. Pedersen SD, Kang J, Kline GA: Portion control plate for weight loss in obese patients with type 2 diabetes mellitus: a controlled clinical trial. Arch Intern Med 2007, 167(12):1277-1283.

9. Ford AL, Bergh C, Sodersten P, Sabin MA, Hollinghurst S, Hunt LP, Shield JP: Treatment of childhood obesity by retraining eating behaviour: randomised controlled trial. BMJ 2010, 340:b5388.

10. Wadden TA, Foster GD: Behavioral treatment of obesity. Med Clin North Am 2000, 84(2):441-461, vii.

11. Volkmar FR, Stunkard AJ, Woolston J, Bailey RA: High attrition rates in commercial weight reduction programs. Arch Intern Med 1981, 141(4):426-428.

12. Bautista-Castano I, Molina-Cabrillana J, Montoya-Alonso JA, Serra-Majem L: Variables predictive of adherence to diet and physical activity recommendations in the treatment of obesity and overweight, in a group of Spanish subjects. Int J Obes Relat Metab Disord 2004, 28(5):697-705.

13. ChooseMyPlate.gov. [http://www.choosemyplate.gov/]

14. Ogden J: The correlates of long-term weight loss: a group comparison study of obesity. Int J Obes Relat Metab Disord 2000, 24(8):1018-1025.

doi:10.1186/1756-0500-4-346

Cite this article as: Kesman et al:: Portion control for the treatment of obesity in the primary care setting. BMC Research Notes 2011 4:346.

\section{Submit your next manuscript to BioMed Central and take full advantage of:}

- Convenient online submission

- Thorough peer review

- No space constraints or color figure charges

- Immediate publication on acceptance

- Inclusion in PubMed, CAS, Scopus and Google Scholar

- Research which is freely available for redistribution

Submit your manuscript at www.biomedcentral.com/submit
C Biomed Central 